\title{
Theoretical descriptions of EEG activity: application to absence
} seizures

\author{
Frank Marten*1, Serafim Rodrigues ${ }^{2}$, Piotr Suffczynski ${ }^{3}$, Mark Richardson ${ }^{4}$ \\ and John R Terry ${ }^{1}$
}

\author{
Address: ${ }^{1}$ Department of Engineering Mathematics, University of Bristol, Bristol, BS8 1TR, UK, 2UNIC, CNRS, Gif-Sur-Yvette, Paris, 91198 , France, \\ ${ }^{3}$ Department of Biomedical Physics, Warsaw University, Warsaw, Poland and ${ }^{4}$ Institute of Psychiatry, King's College London, London, SE5 8AF, \\ UK \\ Email: Frank Marten* - f.marten@bristol.ac.uk \\ * Corresponding author
}

from Eighteenth Annual Computational Neuroscience Meeting: CNS*2009

Berlin, Germany. 18-23 July 2009

Published: 13 July 2009

BMC Neuroscience 2009, 10(Suppl I):P74 doi:I0.I I86/I47I-2202-I0-SI-P74

This abstract is available from: http://www.biomedcentral.com/I47I-2202/I0/SI/P74

(c) 2009 Marten et al; licensee BioMed Central Ltd.

\section{Introduction}

Absence seizures are a class of primary generalized seizures, which typically affect children and young adults. Electroencephalography (EEG) recordings of patients with absence seizures display 2-4 Hz rhythmic activity, the classically observed rhythm being a discharge with a sharp spike and slow wave. However, a systematic study of data from a number of subjects with absence seizures also revealed poly-spike and wave, wave-spike or even no discernable spike-wave onset during seizure events. We present a unifying mathematical framework to study the mechanisms underlying these EEG signals.

\section{Methods}

The model we introduce is a cortico-thalamic system, used to describe the brain's electrical activity as recorded via EEG. The bifurcation structure of this model has been analyzed with the software package MATCONT. The aim of our analysis was to identify parameters that are crucial for the onset of abnormal activity, and investigate mechanisms leading to (poly)spike wave solutions.

\section{Results}

We identified regions in parameter-space where our model supports (poly)spike wave activity. Transitions into these regions occur through Hopf bifurcations, and also through bistability. Hence, our model incorporates two mechanisms to simulate the onset of seizures. Moreover, we investigate the onset of poly-spike wave oscillations; these solutions are created through inflectionpoints, which depend on inhibitory synaptic timescales.

\section{Discussion}

By studying the transitions in a theoretical model for EEG, using a numerical bifurcation analysis, we have identified parameters and mechanisms leading to the onset of (poly)spike wave dynamics. Future work will include a comparison between model and data, by means of parameter fitting. In addition, we aim to enhance our modelling approach by including spatial extent, physiological effects such as neurotransmitter timescales and anatomical effects such as volume conduction. 